\title{
Spatial patterns of genetic variation generated by different forms of dispersal during range expansion
}

\author{
KAMAL M. IBRAHIM $†$, RICHARD A. NICHOLS $\ddagger$ \& GODFREY M. HEWITT ${ }^{*} \dagger$ \\ $\nmid$ School of Biological Sciences, University of East Anglia, University Plain, Norwich NR4 7TJ and $\ddagger$ School of \\ Biological Sciences, Queen Mary and Westfield College, University of London, Mile End Road, London E1 4NS, U.K.
}

\begin{abstract}
We examined the impact of three forms of dispersal, stepping-stone, normal and leptokurtic, on spatial genetic structure of expanding populations using computer simulations. When dispersal beyond neighbouring demes is allowed, rare long-distance migration leads to the establishment of pocket populations in advance of the main invasion front and results in spatial clustering of genotypes which persists for hundreds of generations. Patchiness is more pronounced when dispersal is leptokurtic as is the case in many animal and plant species. These results are of particular interest because population genetic parameters such as gene flow and effective population size are commonly estimated using gene frequency divergence information assuming equilibrium conditions and island models. We show how the three forms of dispersal during colonization bring about contrasting population genetic structures and how this affects estimates of gene flow. The implications for experimental studies of the spatial dimension of population genetic structure are discussed.
\end{abstract}

Keywords: colonization, dispersal, founder events, gene flow, population structure, simulation.

\section{Introduction}

The genetic constitution of most forms of life is not homogeneous in space; it exhibits geographical patterns either because of adaptive differences from place to place or because the homogenizing forces which otherwise smooth out random irregularities have not been effective. One such homogenizing force is the dispersal of organisms throughout the habitat suitable for the species' survival. In population genetics, dispersal is generally viewed as the movement of individuals between demes.

Spatially clustered allele distributions can arise by chance even within continuous populations and in the absence of barriers to dispersal and selection, when only few individuals move between demes and dispersal distance is relatively short (Rohlf \& Schnell, 1971; Endler, 1977; Crow \& Aoki, 1982). Furthermore, occasional extinction and recolonization and the resultant bottlenecks can enhance the rate of differentiation between demes (Wright, 1977). However, Slatkin $(1985,1987)$ has pointed out that, even in the absence of migration, if the

\footnotetext{
${ }^{*}$ Correspondence
}

average time to extinction of local populations is less than the effective population size, extinction and recolonization may actually prevent the genetic differentiation of local populations because of drift. Wade \& McCauley (1988), on the other hand, have shown that differentiation depends critically upon how colonizing groups of individuals are formed and upon the quantitative relationship between the number of colonists and the number of migrants. They argue that in most cases the general effect of extinctions and recolonizations is to promote local genetic differentiation as originally discussed by Wright (1977). More recently, Whitlock \& McCauley (1990) further investigated this using inclusive models which account for the range of scenarios in which founding groups can be formed as well as for the interaction between additive and nonadditive genetic variance (Whitlock et al., 1993).

In deriving these conclusions, Slatkin (1977) and Wade \& McCauley (1988) investigated four models of population structure which differed in the source of the migrant individuals and the colonists. In these models, migration occurs at a constant rate $m$ and migrants are either from an external source with a constant gene frequency or are drawn at random 
from a finite number of subdivided populations. Similarly, new populations are founded by choosing randomly $k$ colonists either from a single randomly chosen local population (the propagule pool model) or the entire metapopulation (the migrant pool model).

These idealized models do not take into account the impact of forms of dispersal, i.e. the spatial distribution of migrants and colonists relative to their source demes, during colonization. In fact, in most analytical and simulation studies of isolationby-distance or drift, forms of dispersal that do not take long-distance movement into account have been assumed (Slatkin, 1981, 1993; Sokal et al., 1989; Epperson, 1993). It is argued that because the numbers of long-distance migrants are small, they are unlikely to become established at their destinations and bring about long-distance gene flow. Rohlf \& Schnell (1971) used simulations to compare dispersal represented by a normal function and stepping-stone-type dispersal but found little difference in the levels of genetic differentiation between localities. However, it has been suggested that the form of dispersal could have a direct effect on spatial genetic structure (Waser \& Elliot, 1991) especially when a species is expanding its range (Larson, 1984; Hewitt, 1993; Templeton et al., 1995).

In many species of plants and animals dispersal is leptokurtic (Bateman, 1950; Aikman \& Hewitt, 1972; Endler, 1977; Lidicker \& Patton, 1987; Waser \& Elliot, 1991). Hence, relative to the distances expected if dispersals are normally distributed, many individuals disperse short distances, few intermediate distances and more long distances. An analytical treatment of the effects of such kurtotic movement using realistic models is highly intractable. Instead, we have used simulations to examine the effects of three forms of dispersal, stepping-stone, normal and leptokurtic, on spatial genetic structure of expanding populations. The impact of this on the estimates of various genetic and demographic parameters will be discussed.

\section{Methods}

The simulation model was implemented in Borland Pascal and run on an IBM-compatible, INTEL 80486-based PC. Two diallelic loci in a diploid organism represented the genetic system. The simulation space comprised a rectangular array of $160 \times 80$ demes each of which was assigned a carrying capacity of 80 individuals. For each deme, the number of individuals and the frequency of each of the four gametic types was stored. Simulations were initiated with all colonized demes populated at carrying capacity $(N=80)$.

When simulating range expansion, eight columns of demes starting at the western (left) edge were inhabited, again at carrying capacity. All allele frequencies were set to 0.5 with no gametic phase disequilibrium. Maximum recombination (0.5) between the two loci. was allowed. The program allows for selection at either of the two loci but we report here only on the case for neutral loci. The sequence of dispersal-growth-random mating was repeated in each deme for a specified number of nonoverlapping generations. Where dispersal forms were compared, the same set of random numbers was used whereas in the case of replicate runs the random number generator (Press et al., 1990) was seeded differently for each run.

\section{Dispersal}

The number of dispersers from each deme was drawn from a binomial distribution with expectation $m$ (migration rate $=0.05$ ) in $N_{t}$ (the number of adults at generation $t$ ) trials. The displacement of each disperser was determined by either a leptokurtic or a normal probability function of mean zero and variance, $v=5$ (Fig. 1). The leptokurtic function was generated by patching the peak of a normal curve with small variance $(\mathrm{N}[0,2])$ and the tail of one with large variance $(\mathrm{N}[0,40)]$ such that the variance of the resultant curve, represented by $(1-a) \mathrm{N}[0,2]+a \mathrm{~N}[0,40]$, was 5 . The ratio in which these two curves were combined was 0.05:0.95. Normal dispersal was represented by the curve $\mathrm{N}[0,5]$.

In the case of stepping-stone dispersal, only neighbouring demes exchanged dispersers, again at a rate $m$. In all cases, two displacement values from the dispersal function were either added or subtracted at random to/from the coordinates of the current deme of each disperser to obtain the coordinates of its destination deme. The simulation space was 'rolled' to avoid top and bottom edge effects. Hence dispersal was possible in all directions except out of the eastern and western edges of the simulation space.

\section{Growth}

The total number of adults in a deme at generation $t\left(N_{t}\right)$ after dispersal was the sum of the nondispersers and the immigrants. The total number of offspring at generation $t+1\left(N_{t+1}\right)$ was drawn from a Poisson distribution with a mean $N_{t+1}^{\prime}=N_{t}+$ $r N_{t}\left(\mathrm{k}-N_{t}\right) / k$, where $k$ is the carrying capacity of the 


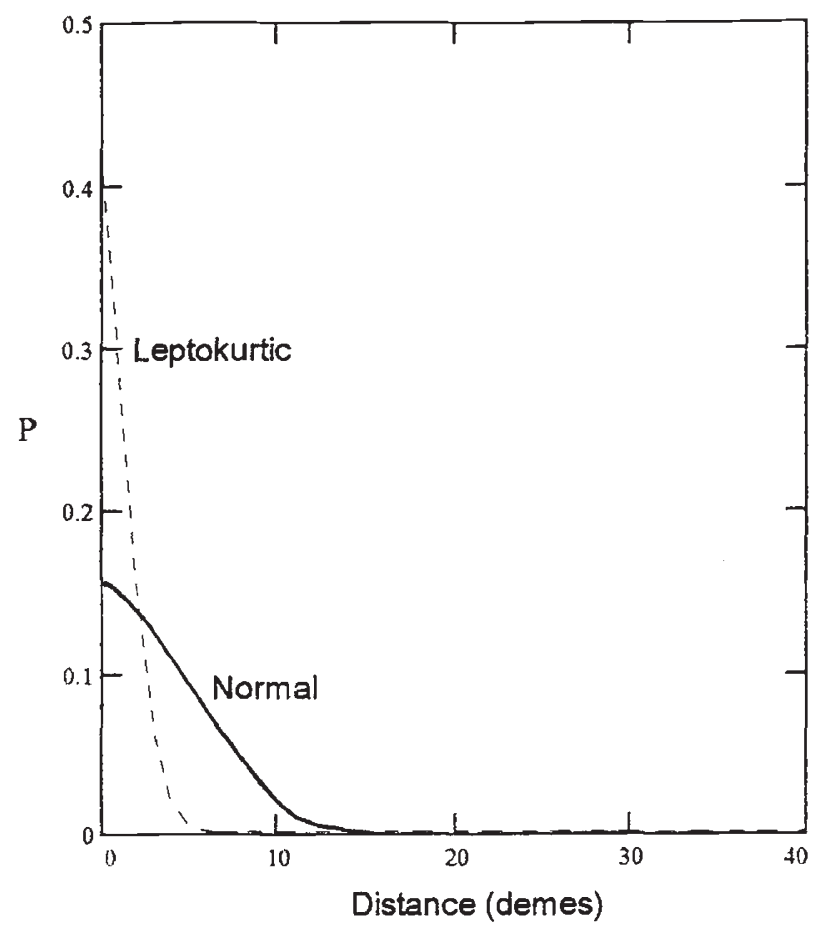

Fig. 1 The leptokurtic and normal dispersal curves used to determine the displacement of each migrant from its deme of origin. Distance is given in demes. Both curves have equal variance, $v=5$ (see text).

deme and $r$ is the intrinsic rate of growth. For all the simulations $r$ was kept constant (0.8); it generates

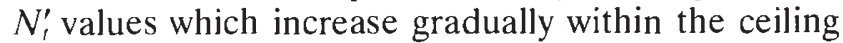
of $k$. $N_{t}$ however, can exceed $k$ if the number of immigrants into a deme is greater than the number of dispersers from the deme producing a stable limit cycle. At the beginning of each simulation, $N_{1}$ is equal to $k$ and zero for inhabited and empty demes, respectively.

\section{Random mating}

In each generation, gametic frequencies and disequilibria in the progeny were calculated as in Falconer (1981). Each offspring was assigned two gametes at random from a rectangular distribution of the frequencies of the four gametic types in the parent population. Because both loci were assumed to be neutral in the present simulations, there was no need to keep track of individual genotypes. Only gametic frequencies were stored.

\section{Sampling}

Population size (mean number of individuals per deme \pm SE) was monitored in successive generations until it reached equilibrium size. Thereafter, allele frequencies at the two loci and $N$, the population size, were saved in successive generations in each and every deme. Contour maps of allele frequencies were drawn using MATHCAD 5.0 (MathSoft, 1994) and an appropriately redimensioned BIOSYs-1 (Swofford \& Selander, 1981) was used for the hierarchical analysis of variance in allele frequency.

\section{Results \\ Dynamics of population size and allele frequency}

The three forms of dispersal reached an equilibrium population size of $76 \pm 6$ individuals per deme by the 80 th generation $(t=80)$. For normal and leptokurtic dispersal, all the demes were colonized by $t=45$, but population size did not stabilize until $t=70$ and $t=80$, respectively. In the case of stepping-stone dispersal, equilibrium population size was reached much quicker because the simulation was initiated with all demes occupied.

The contour maps (Fig. 2) show the spatial distribution of levels of allele frequency at one of the two loci for the three modes of dispersal after 100 and 600 generations. The four colours, red, yellow, green and blue, represent frequency intervals from $\leq 0.25$ to $\geq 0.75$. Stepping-stone dispersal generated a fragmented patchwork of high and low frequency areas whose positions persisted for hundreds of generations but which became more and more fragmented with time (Fig. 2c). Endler (1977) has discussed the implications of these relatively stable clines resulting from stochastic processes and how they could be erroneously ascribed to the action of selective forces in samplebased field studies.

The patches are larger in area and persist longer when dispersal beyond the neighbouring demes is allowed, i.e. in the cases of normal and leptokurtic dispersal. The deviation from the initial 50 per cent allele frequency in these patches is highest for leptokurtic dispersal (Fig. 2a). This could be because, in this form of dispersal, rare long-distance migrants colonize demes way ahead of the main front and act as foci for the establishment of areas of similar genotype derived from a single or a few colonists. We tested this using the data set represented in the form of a contour map as in Fig. 2 in order to see if demes closer to each other have similar allele frequency distributions compared to those further apart. Five hundred pairs of demes, $d$ demes apart $(0<d<16$; for neighbouring demes $d=1)$ were selected at random. Spearman's rank correlation 
Generation 100
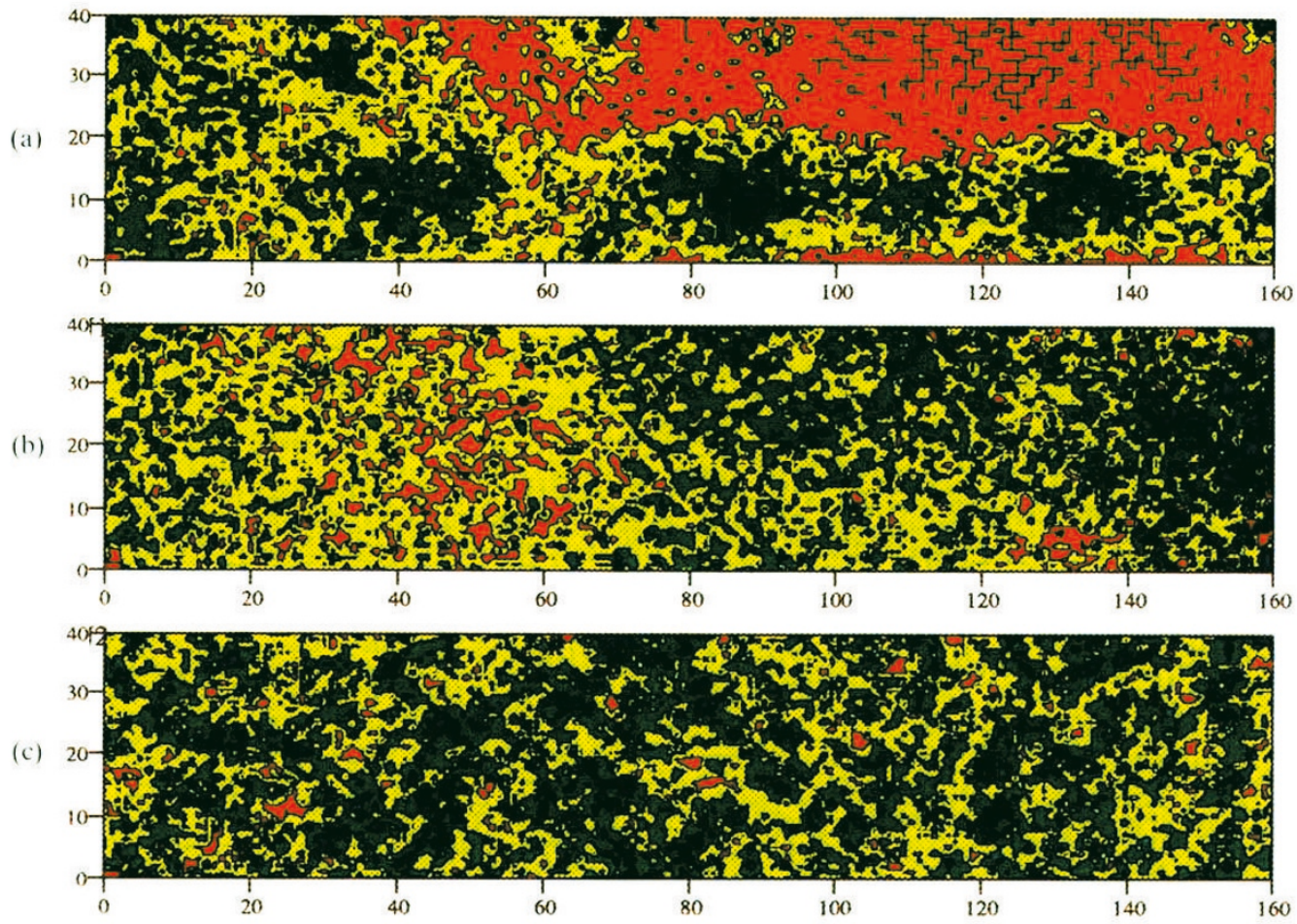

N3

\section{Generation 600}
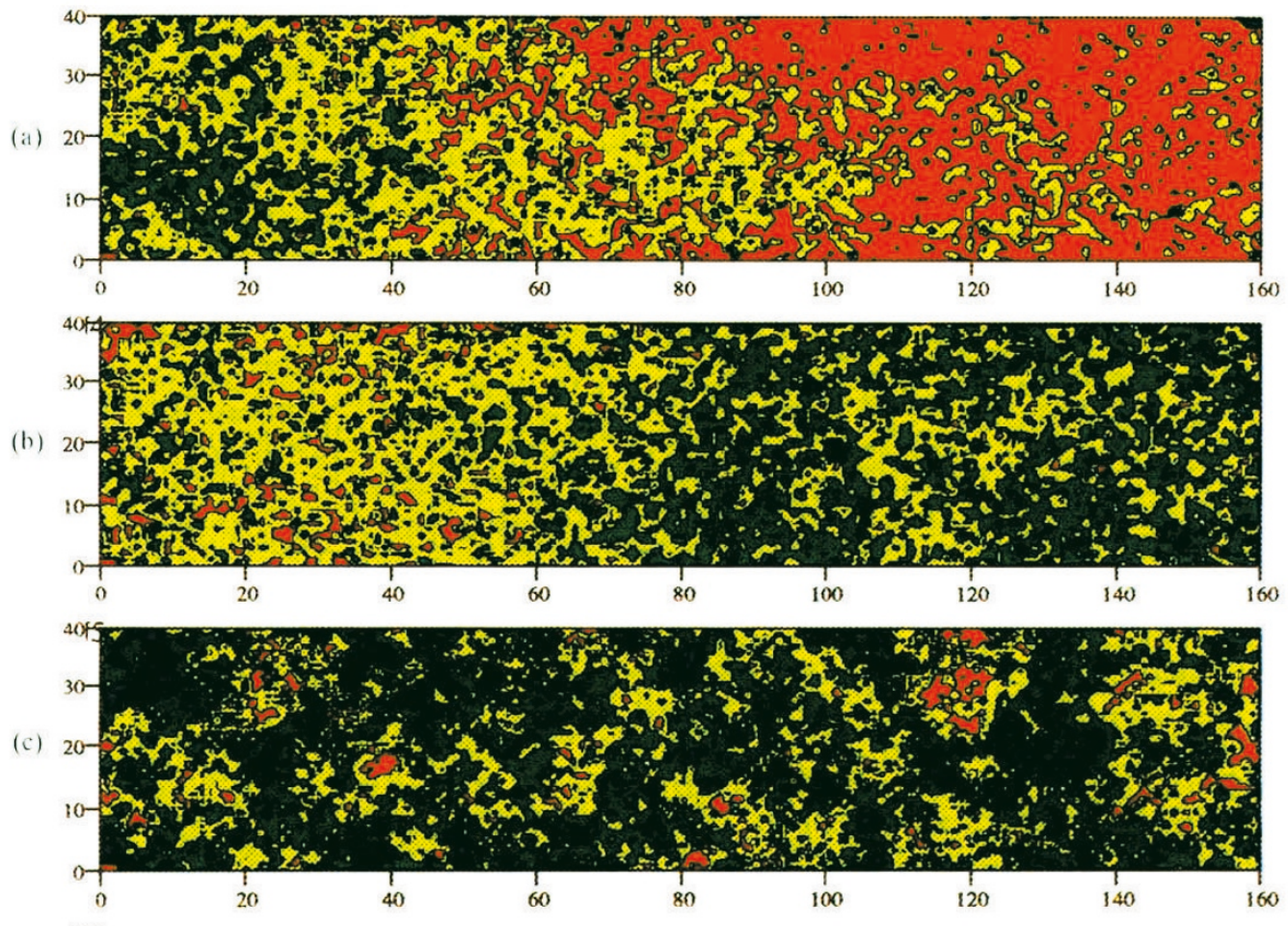

Fig. 2 Contour maps showing the spatial distribution of allele frequency generated by leptokurtic (a), normal (b) and stepping-stone (c) dispersal, at one of the two diallelic loci. Variance in allele frequency within demes and among demes within regions stabilizes at $t=100$ and $t=600$, respectively (see text and Fig. 4). Four levels at intervals of 0.25 are mapped. The four colours, red, yellow, green and blue, represent frequency intervals of $\leq 0.25$ to $\geq 0.75$. 
Generation 100

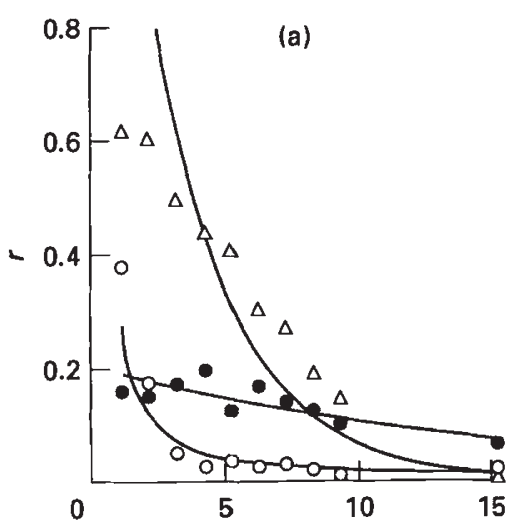

(b)

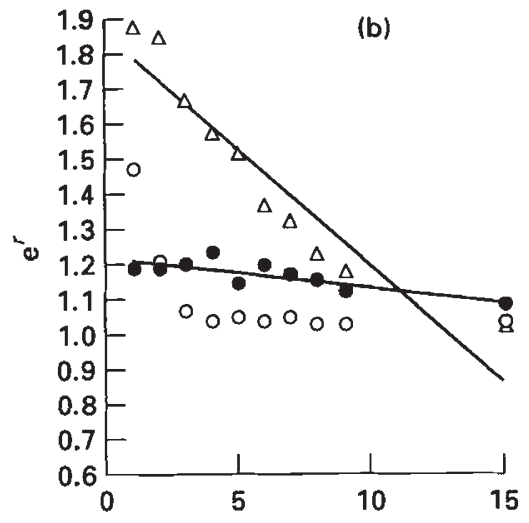

Generation 600
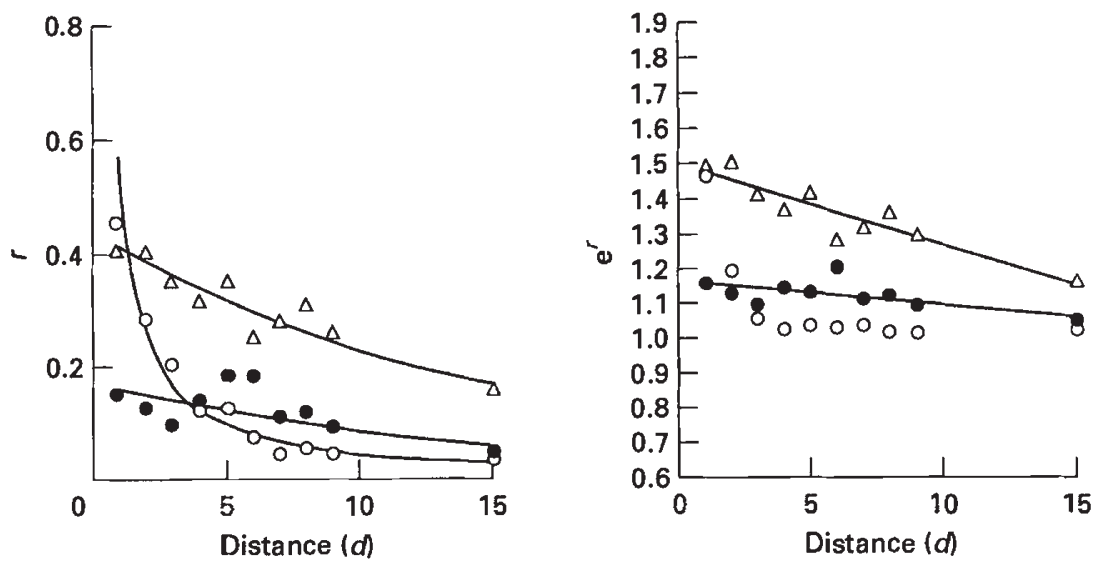

○ Stepping stone $\Delta$ Leptokurtic $\bullet$ Normal

Fig. 3 (a) Spearman's rank correlation coefficient $(r)$ between the allele frequencies in pairs of populations, $d$ demes apart, plotted against the distance, $d$. (b) The same plot with $r$ transformed to $e^{r}$ to show the exponential decline in correlation with distance for leptokurtic and normal dispersal. coefficient $(r)$ between the allele frequencies in the paired demes was computed. An estimate of mean correlation was obtained from six replicate $r s$ for each distance class.

The plot of mean $r$ vs. $d$ (Fig. 3a) clearly shows that: (a) in stepping-stone dispersal the correlation in allele frequency is between neighbouring demes only; (b) there is significant positive correlation $(P<0.01)$ in the allele frequency of demes up to twice the variance of the dispersal curves apart $(10$ demes) in the case of both normal and leptokurtic dispersal; (c) $r$ is consistently higher in leptokurtic dispersal than normal dispersal; (d) there is an exponential decline in $r$ with increase in the distance between the paired demes, and the rate of decline in leptokurtic dispersal is three time as high as that for normal dispersal (Fig. 3b); and (e) $r$ is still significant for distances up to 10 demes after 600 generations; however, the rate of decline of $r$ with distance is much lower. Similar trends were observed in paired demes sampled from $t=200$ and $t=500$. These observations show that the three forms of dispersal generate significantly different spatial distributions of alleles. Long-distance dispersal leads to the clustering of demes with similar allelic frequencies, possibly because of founder effects. This is much more the case when the long-distance dispersal is leptokurtic.

\section{Population genetic structure}

None of the three forms of colonization produced clines or smooth patterns in allele frequency that span the whole simulation space (Fig. 2). The high correlation between the distribution of alleles and the distance between demes, however, indicates the formation of patches or clusters of demes with similar allelic frequencies.

(C) The Genetical Society of Great Britain, Heredity, 77, 282-291. 
Table 1 Components of gene diversity from the heirarchical analysis of the total variance in allele frequency: variance within demes*, among demes within region†, among demesł, among regions§ and totalף

\begin{tabular}{|c|c|c|c|c|c|c|c|}
\hline \multirow[b]{2}{*}{ Generation } & \multirow[b]{2}{*}{ Dispersal } & \multirow[b]{2}{*}{ Replicate } & \multicolumn{5}{|c|}{ Variance components } \\
\hline & & & $H_{\mathrm{D}}^{*}$ & $D_{\mathrm{DR}}{ }^{\dagger}$ & $D_{\mathrm{DT}} \ddagger$ & $D_{\mathrm{RT}} \S$ & $H_{\mathrm{T}} \pi$ \\
\hline \multirow[t]{15}{*}{100} & \multirow[t]{5}{*}{ Leptokurtic } & 1 & 0.2240 & 0.0276 & 0.0909 & 0.0633 & 0.3148 \\
\hline & & 2 & 0.2771 & 0.0507 & 0.2184 & 0.1677 & 0.4955 \\
\hline & & 3 & 0.3002 & 0.0548 & 0.2357 & 0.1809 & 0.5359 \\
\hline & & 4 & 0.2355 & 0.0404 & 0.2812 & 0.2408 & 0.5167 \\
\hline & & Mean & 0.2592 & $\begin{array}{c}0.0434 \\
(0.1413)\end{array}$ & 0.2065 & $\begin{array}{c}0.1632 \\
(0.3504)\end{array}$ & 0.4657 \\
\hline & \multirow[t]{5}{*}{ Normal } & 1 & 0.5959 & 0.1180 & 0.1935 & 0.0755 & 0.7894 \\
\hline & & 2 & 0.5048 & 0.0860 & 0.1594 & 0.0734 & 0.6642 \\
\hline & & 3 & 0.6988 & 0.1138 & 0.1504 & 0.0366 & 0.8492 \\
\hline & & 4 & 0.6917 & 0.1295 & 0.2068 & 0.0773 & 0.8985 \\
\hline & & Mean & 0.6228 & $\begin{array}{c}0.1118 \\
(0.1520)\end{array}$ & 0.1775 & $\begin{array}{c}0.0657 \\
(0.0821)\end{array}$ & 0.8003 \\
\hline & \multirow{5}{*}{$\begin{array}{l}\text { Stepping- } \\
\text { stone }\end{array}$} & 1 & 0.5175 & 0.0806 & 0.1506 & 0.1000 & 0.6680 \\
\hline & & 2 & 0.5154 & 0.0947 & 0.2655 & 0.1708 & 0.7809 \\
\hline & & 3 & 0.5750 & 0.1092 & 0.2940 & 0.1848 & 0.8690 \\
\hline & & 4 & 0.5893 & 0.1183 & 0.2657 & 0.1474 & 0.8550 \\
\hline & & Mean & 0.5493 & $\begin{array}{c}0.1007 \\
(\mathbf{0 . 1 5 5 0})\end{array}$ & 0.2439 & $\begin{array}{c}0.1508 \\
(0.1901)\end{array}$ & 0.7932 \\
\hline \multirow{15}{*}{600} & \multirow{5}{*}{ Leptokurtic } & 1 & 0.2969 & 0.0430 & 0.0977 & 0.0547 & 0.3946 \\
\hline & & 2 & 0.3734 & 0.0418 & 0.1799 & 0.1382 & 0.5534 \\
\hline & & 3 & 0.4591 & 0.0556 & 0.0874 & 0.0318 & 0.5464 \\
\hline & & 4 & 0.2902 & 0.0470 & 0.1965 & 0.1495 & 0.4867 \\
\hline & & Mean & 0.3549 & $\begin{array}{c}0.0468 \\
(0.1190)\end{array}$ & 0.1404 & $\begin{array}{c}0.0936 \\
(0.1890)\end{array}$ & 0.4953 \\
\hline & \multirow[t]{5}{*}{ Normal } & 1 & 0.6254 & 0.1062 & 0.1536 & 0.0474 & 0.7788 \\
\hline & & 2 & 0.5096 & 0.1210 & 0.1830 & 0.0620 & 0.6926 \\
\hline & & 3 & 0.7241 & 0.1220 & 0.1438 & 0.0218 & 0.8679 \\
\hline & & 4 & 0.7051 & 0.1341 & 0.1917 & 0.0576 & 0.8968 \\
\hline & & Mean & 0.6410 & $\begin{array}{c}0.1208 \\
(0.1600)\end{array}$ & 0.1680 & $\begin{array}{c}0.0472 \\
(0.0583)\end{array}$ & 0.8090 \\
\hline & \multirow{5}{*}{$\begin{array}{l}\text { Stepping- } \\
\text { stone }\end{array}$} & 1 & 0.5432 & 0.0547 & 0.1109 & 0.0562 & 0.6540 \\
\hline & & 2 & 0.5525 & 0.0850 & 0.1951 & 0.1101 & 0.7475 \\
\hline & & 3 & 0.7722 & 0.1085 & 0.2003 & 0.0918 & 0.8502 \\
\hline & & 4 & 0.8220 & 0.1084 & 0.2003 & 0.0918 & 0.9139 \\
\hline & & Mean & 0.6725 & $\begin{array}{c}0.0891 \\
(0.1370)\end{array}$ & 0.1766 & $\begin{array}{c}0.0875 \\
(0.1106)\end{array}$ & 0.7914 \\
\hline
\end{tabular}

The figures within parentheses are mean $F_{\mathrm{DR}}$ and $F_{\mathrm{ST}}$, respectively.

In order to test if the three forms of dispersal generated different levels of patchiness, the distribution of alleles in 12 regularly spaced regions (windows) in the simulation space each of $3 \times 4$ demes was saved at $t=100$ and $t=600$. Four replicate simulations were run for each form of dispersal. A hierarchical analysis of the variance in allele frequency (gene diversity) at both loci enabled partitioning the total variance into: within demes $\left(H_{\mathrm{D}}\right)$, between demes within regions $\left(D_{\mathrm{DR}}\right)$, between demes $\left(D_{\mathrm{DT}}\right)$ and between regions $\left(D_{\mathrm{RT}}\right)(\mathrm{Nei}, 1973$,
1977; Wright, 1978). Table 1 shows the components of the total variance for the four replicate runs and estimates of the extent of differentiation between the regions (windows) based on Wright's hierarchical $F$-statistics (Wright, 1965).

The variance within demes, $H_{\mathrm{D}}$, and the total variance, $H_{\mathrm{T}}$, are consistently lowest for leptokurtic dispersal compared to normal and stepping-stone dispersal. The last two generate similar levels of within-deme diversity. Because of the longer range of the rare leptokurtic migrants, they would tend to 
have a longer time to reproduce and fill the demes they colonize before the main front of advance reaches the new habitat. This results in low withindeme variance. The similarity in the levels of $H_{\mathrm{D}}$ generated by normal and stepping-stone dispersal confirms previous findings (Rohlf \& Schnell, 1971). In all four replicate runs and for all forms of dispersal $H_{\mathrm{D}}$ increases with time whereas $H_{\mathrm{T}}$ is more or less at equilibrium.

The variance between the 12 demes within each region, $D_{\mathrm{DR}}$, is also lowest in leptokurtic dispersal. As was evident from the correlation between allele frequency within demes and the distance between demes (Fig. 3), it appears that the bottleneck effect of long-distance colonization has led to the formation of clusters of demes with similar allele frequencies at both loci. It is also possible that the demes within each region show less variability when dispersal is leptokurtic simply because there is more homogenization between all the demes in the simulation space. However, Table 1 shows that the overall variance between demes, $D_{\mathrm{DT}}$, is comparable in all the three forms of dispersal.

Consistent with the expectation from a patchy distribution of alleles and the above observations, the variance between the regions, $D_{\mathrm{RT}}$, is highest for leptokurtic dispersal (Table 1). This is even more evident in the ratio $D_{\mathrm{RT}} / H_{\mathrm{T}}$ (Fig. $4 \mathrm{~b}$ ) which is equivalent to Wright's $F$ measure of subdivision, in this case $F_{\mathrm{RT}}$. After 1500 generations, $F_{\mathrm{RT}}$ is still significantly higher for leptokurtic dispersal, although the rate of decay appears to be faster than in the other forms of dispersal. The measure of differentiation between demes within a region, $F_{\mathrm{DR}}$, is in contrast lowest for leptokurtic dispersal (Fig. 4a). As the simulations progress, intermixing between neighbouring demes increases and $F_{\mathrm{DR}}$ approaches a similar equilibrium value in the three forms of dispersal.

\section{Discussion}

The results presented herein are an attempt at examining the sorts of spatial pattern that can be generated by the combination of mode of dispersal during colonization and drift in two dimensions. The study can be seen as an extension of previous work (Slatkin, 1977; Wade \& McCauley, 1988; Whitlock \& McCauley, 1990) on extinction and recolonization, with special emphasis on the spatial dimension of the impact of pioneer colonization on subsequent population genetic structure.

The metapopulation model used in this study is similar to Slatkin's Model II which comprises a finite

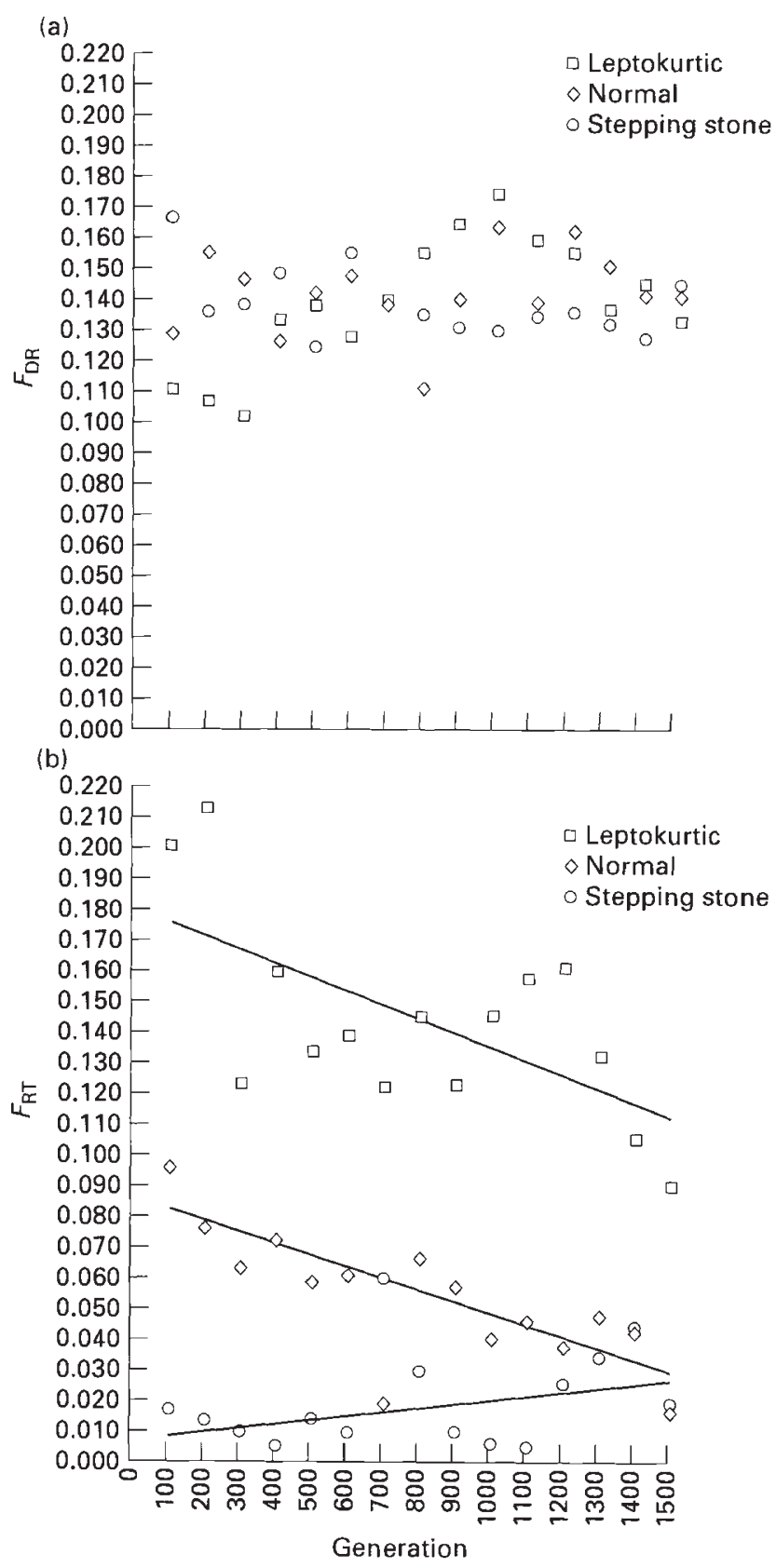

Fig. 4 Measures of differentiation among demes within regions, $F_{\mathrm{DR}}(\mathrm{a})$, and among regions, $F_{\mathrm{RT}}(\mathrm{b})$, plotted against time in generations. When long-distance dispersal is allowed, $F_{\mathrm{DR}}$ values for leptokurtic and normal dispersal approach similar values by $t=600$ whereas $F_{\mathrm{R} T}$ is significantly higher for the former even at $t=1500$.

array of subdivided populations. However, the dispersal functions which define the way in which colonists are chosen to found new populations are quite different from the two forms considered by Slatkin. His 'migrant pool' and 'propagule pool' models, although ideal for analytical investigations, can only represent the extreme ends of the contin- 
uum which exists in nature (Slatkin \& Wade, 1978). Wade \& McCauley (1988) point out that 'migration assortative by distance', where colonies are founded by individuals drawn from nearby populations and not by random samples from the entire metapopulation, is likely to be the common phenomenon in nature. Our normal and leptokurtic models are therefore more realistic representations of the continuum in nature and, all other parameters being equal, would bring about levels of drift-induced genetic differentiation intermediate between the propagule pool and the migrant pool models.

Another distinction is that in Slatkin's models, the new populations founded by $k$ colonists grow to population size $N$ in a single generation and remain at this size until extinction. Our models include logistic growth in population size to an upper limit defined by a carrying capacity. Populations founded by few colonists require several generations to reach the carrying capacity and can exchange migrants during this period. These complexities have precluded an analytical derivation of defined relationships between measures of population genetic structure and the forms of colonization and range expansion modelled in this study. However, the spatial trends observed in several replicated simulations have enabled us to draw some useful conclusions.

As in previous studies (Mollison, 1977; Nichols \& Hewitt, 1994), we have demonstrated that in contrast to the outcome of a steady wave of colonization, rare long-distance migration during range expansion leads to the establishment of pocket populations well in advance of the main wave. The questions of interest are: (a) what are the spatiogenetic consequences, and (b) how long do they persist? Slatkin (1977) and Wade \& McCauley (1988) have shown that the impact of the colonization process is dependent primarily on the numerical relationship between $k$, the number of colonists founding new populations, and $\mathrm{Nm}$, the number of individuals exchanged between extant populations. Because the pocket populations in the case of normal and leptokurtic dispersal are founded by rare long-distance migrants, they tend to show high levels both of inbreeding within populations (demes) and of differentiation between populations. The lag time between the formation of the advance colonies and the main body of the expanding populations leads to the formation of patches of highly inbred demes founded by migrants from the initial colonies (Fig. 2). This effect is more pronounced in the case of leptokurtic dispersal (Fig. 3, Table 1) because long-distance migrants are more common. Hence, the new colonies formed by these migrants have more time to colonize neighbouring demes before the main invasion front starts contributing to their gene pool. This results in lower within-deme and within-patch differentiation, and higher betweenpatches differentiation compared to the normal model. These basic trends which result from early events during colonization history persist for hundreds of generations (Fig. 2).

To highlight further these trends, we estimated the number of dispersers exchanged between the regions (patches) in our simulation space using Wright's (1969) approximation of the gene flow parameter, $\mathrm{Nm}$. For the island model where an array of populations receive migrants from a genetically constant outside source, and assuming negligible mutation rates, an estimate of $\mathrm{Nm}$ can be obtained using the approximation $N m=\left(1-F_{\mathrm{ST}}\right) / 4 F_{\mathrm{ST}}$. After 1500 generations (using the values for $F_{\mathrm{RT}}$ shown in Fig. 4b) the estimates were 13.63, 14.45 and 2.38 individuals per generation for normal, steppingstone and leptokurtic dispersal, respectively. This shows that apparently identical dispersal as characterized by the variance in parent-offspring distance (Hengeveld, 1989, pp. 60-62) can lead to the varying spatial distribution of genes. The initial colonization events can explain this paradox. In the case of leptokurtic colonization, rare long-distance migrants have acted as foci for the establishment of patches of similar genotypes derived from a single or a few colonists. Different alleles may have, by chance, become abundant in different patches. The regions being compared fall within the area of these patches and reflect the extent of the differentiation between them. The estimates of local gene flow between the 12 demes within each region were $1.54,1.60$ and 1.67 for stepping-stone, normal and leptokurtic dispersal, respectively, confirming that the regions lie within relatively homogenous patches. To summarize, these estimates reveal that gene frequency differences that arise during founding can persist for hundreds of generations and estimates of present gene flow based on the divergence would be inaccurate.

Poor correlations between indirect estimates of gene flow based on gene frequency differences and the dispersal capability of organisms have been reported in a number of empirical studies (Singh \& Rhomberg, 1987; Liebherr, 1988). Boileau et al. (1992) drew attention to this problem in their allozyme survey of aquatic invertebrate taxa. Their estimates of gene flow from gene frequency divergence were inconsistent with the dispersal capacities of the taxa. They proposed historical events as an alter- 
native explanation and showed that when populations are founded from a few individuals and subsequently undergo rapid growth before exchanging migrants, the founder effect can persist for thousands of generations. This study shows that colonization by rare long-distance migrants can result in a similar population genetic structure.

Similarly, the significance of forms of dispersal in studies that attempt to estimate effective population size, $N_{\mathrm{e}}$, was demonstrated by Waser \& Elliot (1991). Using the simplest approximation of $N_{\mathrm{e}}$ $\left(N_{\mathrm{e}}=4 \pi \sigma^{2} d\right)$, and representing $d$ by the absolute distance between the natal and breeding locations of juveniles, they showed that kurtosis in the distribution of $d$ reduces the $N_{\mathrm{e}}$ estimates of populations of the kangaroo rat, Dipodomys spectabilis.

Finally, these results have obvious implications on the interpretation of the spatial dimension of genetic variation. Endler (1977) has discussed how patchy distributions of neutral alleles could be ascribed to the action of selective forces. Thus, unless effective selection in favour or against particular alleles has been demonstrated as in the classic case of the aminopeptidase cline in the marine bivalve mussel, Mytilus edulis (Koehn et al., 1980, 1983), correlation between the frequency of polymorphic genes and spatial or temporal variation in environmental factors may not be caused by natural selection only.

The action of natural selection can also be tested by comparing the observed variability of the variance in allele frequency among loci with that expected when no natural selection is acting. As pointed out by Easteal $(1988,1989)$, these and other theoretical predictions which are based on static models of population structure are not necessarily valid for populations undergoing range expansion. Therefore, because simple relationships between dispersal and genetic structure of metapopulations may not exist, simulation studies can assist in understanding some aspects of the patterns revealed by experimental data.

\section{Acknowledgements}

We thank the BBSRC for financial support and Nicola Flanagan for stimulating discussions. Helpful suggestions from two anonymous referees are gratefully acknowledged.

\section{References}

AIKMAN, D. AND HEwITT, G. M. 1972. An experimental investigation of the rate and form of dispersal in grasshoppers. J. Appl. Ecol., 9, 867-877.

Bateman, A. J. 1950. Is gene dispersion normal? Heredity,
4, 353-364.

BOILEAU, M. G., HEBERT, P. D. N. AND SCHWARTZ, S. S. 1992. Non-equilibrium gene frequency divergence: persistent founder effects in natural populations. J. Evol. Biol., 5, 25-39.

CROW J. F. AND AOK1, K. 1982. Group selection for a polygenic behavioral trait: A differential proliferation model. Proc. Natl. Acad. Sci. U.S.A., 79, 2628-2631.

EASTEAL, s. 1988. Range expansion and its genetic consequences in populations of the Giant Toad, Bufo marinus. Evol. Biol., 23, 49-84.

EASTEAL, s. 1989. The effect of genetic drift during range expansion on geographical patterns of variation: a computer simulation of the colonization of Australia by Bufo marinus. Biol. J. Linn. Soc., 37, 281-295.

ENDler, J. A. 1977. Geographic Variation, Speciation, and Clines. Princeton University Press, Princeton, NJ.

EPPERSON, B. K. 1993. Recent advances in correlation studies of spatial patterns of genetic variation. Evol. Biol., 27, 95-155.

FAlCONER, D. S. 1981. Introduction to Quantitative Genetics, 2nd edn. Longman, London.

HengeVeld, R. 1989. Dynamics of Biological Invasions, pp. 60-62. Chapman and Hall, London.

HEwITT, G. M. 1993. Postglacial distribution and species substructure: lessons from pollen, insects and hybrid zones. In: Lees, D. R. and Edwards, D. (eds) Evolutionary Patterns and Processes, pp. 97-123. Linnaean Society of London, Academic Press, London.

KOEHN, R. K., NEWELL, R. I. E AND IMMERMANN, F. 1980. Maintenance of an aminopeptidase allele frequency cline by natural selection. Proc. Natl. Acad. Sci. U.S.A., 77, 5385-5389.

KOEHN, R. K., ZERA, A. J. AND HALl, J. G. 1983. Enzyme polymorphism and natural selection. In: Nei, M. and Koehn, R. K. (eds) Evolution of Genes and Proteins, pp. 115-136. Sinauer Associates, Sunderland, MA.

LARSON, A. 1984. Neontological inferences of evolutionary pattern and process in the salamader family Plethodontidae. Evol. Biol., 17, 119-217.

LIEBHERR, J. K. 1988. Gene flow in ground beetles (Coleoptera: Carabidae) of differing habitat preference and flight-wing development. Evolution, 42, 129-137.

LIDICKER, W. Z. AND PATTON, J. L. 1987. Patterns of dispersal and genetic structure in populations of small rodents. In: Chepko-Sade, B. D. and Halpin, Z. T. (eds) Mammalian Dispersal Patterns, the Effects of Social Structure on Population Genetics, pp. 144-161. University of Chicago Press, Chicago, IL.

Mathsoft 1994. MathCad Plus 5.0 for Windows. MathSoft Inc., Cambridge, MA.

MOLL1SsON, D. 1977. Spatial contact models for ecological and epidemic spread. J. R. Statist. Soc. B, 39, 283-326.

NEI, M. 1973. Analysis of gene diversity in subdivided populations. Proc. Natl. Acad. Sci. U.S.A., 70 , 3321-3323.

NEI, M. 1977. F-statistics and the analysis of gene diversity in subdivided populations. Ann. hum. Genet., 41, 225-233. 
NICHOLS, R. A. AND HEWITT, G. M. 1994. The genetic consequences of long distance dispersal during colonization. Heredity, 72, 312-317.

PRESS, W. H., FLANNERY, B. P., TEUKOLSKY, S. A. AND Vetterling, w. T. 1990. Numerical Recipes in Pascal. Cambridge University Press, Cambridge, U.K.

ROHLF, F. J. AND SCHNELL, G. D. 1971. An investigation of the isolation-by-distance model. Am. Nat., 105, 295-324.

SINGH, R. S. AND RHOMBERG, L. R. 1987. A comprehensive study of genic variation in natural populations of Drosophila melanogaster. I. Estimates of gene flow from rare alleles. Genetics, 115, 313-322.

SLATKIN, M. 1977. Gene flow and genetic drift in a species subject to frequent local extinctions. Theor. Pop. Biol., 12, 253-262.

SLATKIN, M. 1981. Estimating levels of gene flow in natural populations. Genetics, 99, 323-335.

Slatkin, M. 1985. Gene flow in natural populations. Ann. Rev. Ecol. Syst., 16, 393-430.

SLATKIN, M. 1987. Gene flow and the geographic structure of natural populations. Science, 236, 787-792.

SLATKIN, M. 1993. Isolation by distance in equilibrium and non-equilibrium populations. Evolution, 47, 264-279.

SLATKIN, M. AND WADE, M. J. 1978. Group selection on a quantitative character. Proc. Natl. Acad. Sci. U.S.A., 75, 3531-3534.

SOKAL, R. R., JACQUEZ, G. M. AND WOOTEN, M. C. 1989. Spatial autocorrelation analysis of migration and selection. Genetics, 121, 845-855.

SWOFFORD, D. L. AND SELANDER, R. B. 1981. BIOSYS-1 a FORTRAN program for the comprehensive analysis of electrophoretic data in population genetics and systematics. J. Hered., 72, 281-283.

TEMPLETON, A. R., ROUTMAN, E. AND PHILlips, C. A. 1995. Separating population structure from population history: a cladistic analysis of the geographical distribution of mitochondrial DNA haplotypes in the tiger salamader, Ambystoma tigrinum. Genetics, 140, 767-782.

WADE, J. W. AND McCAUleY, D. E. 1988. Extinction and recolonization: their effects on the genetic differentiation of local populations. Evolution, 42, 995-1005.

WASER, P. M. AND ELLIOT, L. F. 1991. Dispersal and genetic structure in kangaroo rats. Evolution, 45, 935-943.

WHITLOCK M. C. AND McCAULEY, D. E. 1990. Some population genetic consequences of colony formation and extinction: genetic correlations within founding groups. Evolution, 44, 1717-1724.

WHITLOCK, M. C., PHILliPS, P. C. AND WADE, M. J. 1993. Gene interaction affects the additive genetic variance in subdivided populations with migrations and extinction. Evolution, 47, 1758-1769.

WRIGHT, s. 1965. The interpretation of population structure by F-statistics with special regard to systems of mating. Evolution, 19, 395-420.

WRIGHT, s. 1969. Evolution and the Genetics of Populations, vol. 2, The Theory of Gene Frequencies. University of Chicago Press, Chicago, IL.

WRIGHT, s. 1977. Evolution and the Genetics of Populations, vol. 3, Experimental Results and Evolutionary Deductions. University of Chicago Press, Chicago, IL.

WRIGHT, s. 1978. Evolution and the Genetics of Populations, vol. 4, Variability Within and Among Natural Populations. University of Chicago Press, Chicago, IL. 